\title{
Glucocorticoids in acute pancreatitis: a propensity score matching analysis
}

\author{
Meng Wang ${ }^{1 \dagger}$, Zongxing Jiang ${ }^{2 \dagger}$ and Hongyin Liang ${ }^{2 *}$
}

\begin{abstract}
Background: There are few reports about the effect of glucocorticoids in the treatment of acute pancreatitis in humans. This study aims to evaluate the effect of glucocorticoids in the treatment of acute pancreatitis by propensity score matching analysis.

Results: Acute pancreatitis patients admitted between 2014 and 2019 were collected from the database and analyzed. Included patients were divided into the glucocorticoids-used group (GC group) and the non-glucocorticoids-used group (NGC group) according to whether glucocorticoids were used. A total of 818 eligible patients were included in the final analysis. Seventy-six patients were treated with glucocorticoids, and 742 patients were treated without glucocorticoids. Before propensity score matching, the triglyceride levels (38.2 $\pm 18.5 \mathrm{vs.} 20.2 \pm 16.8$, $P<0.05)$ and Acute Physiology and Chronic Health Evaluation II (APACHE II) scores $(7.1 \pm 2.5$ vs. $4.5 \pm 2.1, P<0.05)$ at admission were significantly higher in the GC group than in the NGC group. The incidence of multi-organ failure (33.3\% vs. $11.9 \%, P<0.05$ ) was significantly higher in the GC group than in the NGC group. Patients in the GC group showed a positive balance of fluid intake and output over $72 \mathrm{~h}$. After 1:1 propensity score matching, 59 patients from each group (GC and NGC) were included in the analysis. There were no significant differences in age, sex, body mass index, triglycerides, or APACHE II scores between the two groups $(P>0.05)$, and the patients' clinical outcomes were reversed. The proportion of patients with organ failure $(40.7 \%$ vs. $52.5 \%, p<0.05)$ and multi-organ failure $(35.0 \%$ vs. $67.7 \%, P<0.05)$ was significantly lower in the GC group than in the NGC group. Furthermore, patients in the GC group had significantly shorter lengths of hospital stay $(12.9 \pm 5.5 \mathrm{vs}$. $16.3 \pm 7.7, P<0.05)$ and costs $(25,348.4 \pm 2512.6 \mathrm{vs}$. $32,421.7 \pm 2813.3, P<0.05)$ than those in the NGC group.
\end{abstract}

Conclusions: This study presents preliminary confirmation of the beneficial effect of glucocorticoids in the treatment of acute pancreatitis. More high-quality prospective studies are needed in the future.

Keywords: Glucocorticoids, Acute pancreatitis, Hypertriglyceridemic, Propensity score matching analysis

\section{Introduction}

Acute pancreatitis (AP) is a commonly occurring acute abdominal disease with multiple causes [1]. Severe acute pancreatitis (SAP) may be life-threatening and result in serious economic and health burdens [2]. During the

\footnotetext{
*Correspondence: lianghy1212@126.com

${ }^{\dagger}$ Meng Wang and Zongxing Jiang have contributed equally to this work. 2 Department of General Surgery, General Hospital of Western Theater Command (Chengdu Military General Hospital), Chengdu 613000, China Full list of author information is available at the end of the article
}

early stage of AP, the systemic inflammatory response and subsequent multi-organ impairment are the most significant manifestations, resulting in the first peak of death in patients with AP $[3,4]$.

Glucocorticoids (GCs) are broad-spectrum inflammation-suppressing drugs that exert a broad range of anti-inflammatory effects [5]. GCs are versatile and may lead to some adverse effects, such as hyperglycaemia, secondary infections, osteoporosis, wound healing difficulty, and gastrointestinal bleeding [6]. At present, the effects of GCs in asthma, chronic obstructive pulmonary 
disease (COPD), and systemic vasculitides are wellacknowledged [7]. GCs are also considered to have beneficial, though debatable, effects in a series of other inflammatory diseases, including sepsis shock [8], community-acquired pneumonia [9], burns [10], and acute respiratory distress syndrome (ARDS) [11]. Recently, it has been reported that patients with COVID-19 may also benefit from the use of GCs to counter inflammatory storms [12, 13].

The role of GCs in the treatment of AP has been studied for a long time $[14,15]$. In animal models of AP, the use of GCs has shown favorable results [16, 17]. However, there are few reports about the effect of GCs in the treatment of AP in humans. In this study, we used the propensity score matching (PSM) analysis to re-examine the contribution of GCs in the treatment of AP.

\section{Materials and methods}

\section{Study design}

This is a retrospective single-center cohort study. This study was performed following approval from the ethics committee of the General Hospital of Western Theater Command (GGW2019017) and conducted in accordance with the Declaration of Helsinki.

We utilized a database that recorded the information of AP patients admitted to our hospital since 2012 to conduct this retrospective research. All patients in this database between January 2014 and December 2019 were screened. Eligible patients were 18-70 years old, diagnosed with hypertriglyceridemic AP, and admitted within $48 \mathrm{~h}$ of onset. AP patients complicated with immune deficiency, pregnancy, or malignancy were excluded from this study. Patients included were divided into the glucocorticoids-used group (GC group) and the nonglucocorticoids-used group (NGC group) according to whether GCs were used.

The diagnosis and severity of AP were determined based on the 2012 Atlanta criteria [1]. The identification of hypertriglyceridemic AP was performed according to previous studies, and the serum triglyceride (TG) levels of patients with hypertriglyceridemic pancreatitis were usually above $11.3 \mathrm{mmol} / \mathrm{L}$ [18].

\section{Data collection}

The data were collected from this database of AP patients. All data collectors were blinded to the study aims being investigated at the time of data abstraction. All medical records were reviewed by another independent physician. Inconsistent scores were recalculated until the same scores were achieved.

Demographic information, including age and sex, was collected. Some valuable evaluations were also performed. For example, Acute Physiology and Chronic
Health Evaluation (APACHE II) scores [19] and Sequential Organ Failure Assessment (SOFA) scores [20] were collected to assess the severity of AP. The APACHE II and SOFA scores were calculated using the worst parameters during the initial $24 \mathrm{~h}$ after admission. Serum TGs at admission and body mass index (BMI) were also recorded. The collected therapeutic outcome indicator data included mortality, prevalence, duration of organ failure, the proportion of patients requiring further intervention, the incidence of peripancreatic necrosis infection and gastrointestinal bleeding, length of stay (LOS), and hospital costs. Several indicators were also evaluated to estimate the impact of GCs administration on fluid resuscitation. Inflammatory indicators, such as C-reactive protein (CRP) and tumor necrosis factor- $\alpha$ (TNF- $\alpha$ ), at 1 week after hospitalization were also measured.

\section{Management of AP}

Initially, all patients were treated conservatively as recommended in the guidelines [21, 22]. A revised step-up approach was used to manage AP-associated complications, including ascites, acute peripancreatic fluid collection, acute necrotic collection, and infected necrosis [23]. Prophylactic antibiotics are not routinely utilized. If the patient has symptoms of systemic infection, as well as elevated temperature, increased blood count, or positive blood cultures, antibiotics may be administered as appropriate.

Until now, there is no evidence to support the conventional utilization of GCs in AP. GCs are only used in some AP patients with severe inflammatory responses in the early stages of AP in our center. For example, the patients with hemodynamic instability (mean arterial pressure $\leq 65 \mathrm{~mm} \mathrm{Hg}$ ), severe acute respiratory distress $(\mathrm{PaO} 2 / \mathrm{FiO} 2 \leq 100 \mathrm{mmHg})$, persistent organ failure $(>24 \mathrm{~h})$, or multi-organ failure within the first $48 \mathrm{~h}$ of onset were considered for GCs treatment. The results of such a scheme included: (1) Patients treated with GCs tended to be more severely; (2) Not every patient with severe disease was treated with GCs. Therefore, in this retrospective study, we grouped based only on whether GCs were used. The PSM analysis was used to match the baseline between two groups, and thus further analysis was performed, which is not conducted in previous researches. The major types of GCs used were prednisone, dexamethasone, and hydrocortisone. The duration of GCs used was 1-6 days. The doses of prednisone or the equivalent doses of other glucocorticoids are usually less than $80 \mathrm{mg} / \mathrm{d}$.

\section{Propensity score-matching analysis}

PSM analysis was performed in this study with the matching package in $\mathrm{R}$ software (version 4.0.2 for 
Windows, Bell Laboratories) and conducted with the 1:1 nearest neighbor matching method. The covariates included sex, age, BMI, TGs, APACHE II scores, and SOFA scores.

\section{Statistical analysis}

Categorical variables are described using frequencies and percentages. Continuous variables are summarized as medians (quartiles) or mean values $( \pm$ SDs) when appropriate. Pearson $\chi^{2}$ test or Fisher's exact test was used to determining the association between categorical variables. The student's t-test or the Wilcoxon rank-sum test was used for continuous data as appropriate. Analysis of variance (ANOVA) or the Kruskal-Wallis test was used for comparisons between more than two groups, as appropriate. Statistical analysis was performed using SPSS, version 20.0 for Windows (SPSS Inc., Chicago, IL). $P<0.05$ was considered statistically significant.

\section{Results}

From January 2014 to December 2019, a total of 3478 patients were admitted to our hospital and documented in the database. Among these patients, 818 patients who met the inclusion and exclusion criteria were included in the final analysis. Seventy-six patients were treated with GCs, regardless of the type or amount of GCs used or the duration of GCs use.

The baseline characteristics were matched via PSM analysis The baseline characteristics of the patients are shown in Table 1. Before propensity score matching, the serum TGs $(38.2 \pm 18.5$ vs. $20.2 \pm 16.8, P=0.005<0.05)$ and APACHE II scores $(7.1 \pm 2.5$ vs. $4.5 \pm 2.1, P=0.034<0.05)$ at admission were significantly higher in the GC group than in the NGC group. The SOFA score $(4.4 \pm 1.5$ vs. $2.1 \pm 1.6, P=0.063)$ was higher in the GC group than in the NGC group, although the difference was not statistically significant. These results suggest that patients in the
GC group had a higher severity of disease at admission than those in the NGC group. After 1:1 propensity score matching, 59 patients from each group (GC and NGC) were enrolled in the analysis. There were no significant differences in age, sex, BMI, TGs, APACHE II scores, or SOFA scores between the two groups.

\section{The comparison of the clinical outcomes between two groups}

The clinical outcomes of the two groups are shown in Table 2. Before propensity score matching, there was no difference in mortality between the two groups $(3.9 \%$ vs. $2.4 \%, P=0.676$ ). In the GC group, 2 patients died of severe secondary infection of peripancreatic necrosis and 1 patient died of organ failure in the early stage of AP. In the NGC group, the major cause of death is also the secondary infection of peripancreatic necrosis $(n=11)$. The other cause of death included pulmonary Infection $(n=3)$, peripancreatic necrotic hemorrhage caused by puncture $(n=2)$, and organ failure in late-stage $(n=2)$. The incidence of multi-organ failure was significantly higher in the GC group than in the NGC group (33.3\% vs. $11.9 \%, P=0.003<0.05)$. The incidences of organ failure (31.4\% vs. $24.9 \%, P=0.206)$, organ failure lasting more than $48 \mathrm{~h}(45.8 \%$ vs. $36.2 \%, P=0.124)$, peripancreatic necrotic infection ( $14.5 \%$ vs. $10.1 \%, P=0.324)$, and proportion of SAP $(32.9 \%$ vs. $28.0 \%, P=0.371)$ were higher in the GC group than in the NGC group, but there was no significant difference. However, there was no significant difference in the proportion of patients using antibiotics between the two groups $(51.3 \%$ vs. $46.9 \%$, $P=0.463)$. The proportion of patients requiring advanced interventions, such as abdominal paracentesis drainage (APD), percutaneous catheter drainage (PCD), or open surgery, was not significantly different between the two groups $(P>0.05)$. The LOS in the ICU was significantly higher in the GC group than in the NGC group (3.0 \pm 1.9 vs. $1.2 \pm 0.7, P<0.001)$. Pain duration, fasting time, and

Table 1 Patient Characteristics at Baseline

\begin{tabular}{|c|c|c|c|c|c|c|}
\hline \multirow[t]{2}{*}{ Characteristic } & \multicolumn{2}{|c|}{ Original cohort $(n=818)$} & \multirow[t]{2}{*}{$P$} & \multicolumn{2}{|c|}{ Matched cohort $(n=118)$} & \multirow[t]{2}{*}{$P$} \\
\hline & GC group $(n=76)$ & NGC group $(n=742)$ & & GC group $(n=59)$ & NGC group $(n=59)$ & \\
\hline Age, year & $45.1 \pm 14.3$ & $42.3 \pm 16.6$ & 0.429 & $44.9 \pm 15.3$ & $44.1 \pm 16.1$ & 0.894 \\
\hline Sex, Male, n (\%) & $44(57.89 \%)$ & $385(51.89 \%)$ & 0.132 & $33(55.93 \%)$ & $34(57.62 \%)$ & 0.813 \\
\hline $\mathrm{BMI}, \mathrm{kg} / \mathrm{m}^{2}$ & $28.1 \pm 4.8$ & $25.7 \pm 3.9$ & 0.107 & $27.2 \pm 4.9$ & $27.0 \pm 4.1$ & 0.927 \\
\hline $\mathrm{TGs}, \mathrm{mmol} / \mathrm{L}$ & $38.2 \pm 18.5$ & $20.2 \pm 16.8$ & $0.005^{*}$ & $37.1 \pm 17.9$ & $37.51 \pm 17.75$ & 0.798 \\
\hline APACHE II score & $7.1 \pm 2.5$ & $4.5 \pm 2.1$ & $0.034^{*}$ & $7.2 \pm 2.4$ & $7.1 \pm 2.3$ & 0.829 \\
\hline SOFA score & $4.4 \pm 1.5$ & $2.1 \pm 1.6$ & 0.063 & $4.3 \pm 1.5$ & $4.3 \pm 1.5$ & 0.968 \\
\hline
\end{tabular}

Abbreviation: BMI, Body mass index; TGs, Triglycerides; APACHE II, Acute Physiology and Chronic Health Evaluation II; SOFA, Sequential Organ Failure Assessment *Significant difference 
Table 2 Main Clinical Outcomes of the Study

\begin{tabular}{|c|c|c|c|c|c|c|}
\hline \multirow[t]{2}{*}{ Characteristic } & \multicolumn{2}{|c|}{ Original cohort $(n=818)$} & \multirow[t]{2}{*}{$P$} & \multicolumn{2}{|c|}{ Matched cohort $(n=118)$} & \multirow[t]{2}{*}{$P$} \\
\hline & GC group $(n=76)$ & NGC group $(n=742)$ & & GC group $(n=59)$ & NGC group $(n=59)$ & \\
\hline Mortality, n (\%) & $3(3.9 \%)$ & $18(2.4 \%)$ & 0.676 & $3(5.1 \%)$ & $8(13.6 \%)$ & 0.113 \\
\hline Severe acute pancreatitis, n (\%) & $25(32.9 \%)$ & $208(28.0 \%)$ & 0.371 & $23(39.0 \%)$ & $33(55.9 \%)$ & 0.065 \\
\hline \multicolumn{7}{|l|}{ Organ failure } \\
\hline Patient number, n (\%) & $24(31.6 \%)$ & $185(24.9 \%)$ & 0.206 & $20(40.7 \%)$ & $31(52.5 \%)$ & $0.041^{*}$ \\
\hline \multicolumn{7}{|l|}{ Duration, n (\%) } \\
\hline$<48 \mathrm{~h}$ & $13(54.2 \%)$ & $118(63.8 \%)$ & 0.785 & $10(50.0 \%)$ & $12(38.7 \%)$ & 0.636 \\
\hline$\geq 48 \mathrm{~h}$ & $11(45.8 \%)$ & $67(36.2 \%)$ & 0.124 & $10(50.0 \%)$ & $19(61.3 \%)$ & 0.054 \\
\hline \multicolumn{7}{|l|}{ Organs involved, n (\%) } \\
\hline Single & $16(66.7 \%)$ & $163(88.1 \%)$ & 0.854 & $13(65.0 \%)$ & $10(32.3 \%)$ & 0.486 \\
\hline Mutiple & $8(33.3 \%)$ & $22(11.9 \%)$ & $0.003^{*}$ & $7(35.0 \%)$ & $21(67.7 \%)$ & $0.002^{*}$ \\
\hline \multicolumn{7}{|l|}{ Further interventions needed, $\mathrm{n}(\%)$} \\
\hline APD & $15(19.7 \%)$ & $139(18.7 \%)$ & 0.831 & $11(18.6 \%)$ & $17(28.8 \%)$ & 0.194 \\
\hline PCD & $10(13.2 \%)$ & $72(9.7 \%)$ & 0.340 & $8(13.6 \%)$ & $13(22.0 \%)$ & 0.229 \\
\hline Minimally invasive interventions & $5(6.6 \%)$ & $24(3.2 \%)$ & 0.240 & $4(6.8 \%)$ & $7(11.9 \%)$ & 0.342 \\
\hline Open operation & $2(2.6 \%)$ & $11(1.5 \%)$ & 0.778 & $2(3.4 \%)$ & $3(5.1 \%)$ & 0.648 \\
\hline Antibiotic usage, n (\%) & $39(51.3 \%)$ & $348(46.9 \%)$ & 0.463 & $28(47.5 \%)$ & $29(49.2 \%)$ & 0.854 \\
\hline \multicolumn{7}{|l|}{ Glucocorticoids related adverse events } \\
\hline Infection, n (\%) & $23(30.3 \%)$ & $221(29.8 \%)$ & 0.892 & $19(32.2 \%)$ & $18(30.5)$ & 0.843 \\
\hline Peripancreatic necrosis infection, n (\%) & $11(14.5 \%)$ & $75(10.1 \%)$ & 0.324 & $9(15.3 \%)$ & $15(25.4 \%)$ & 0.170 \\
\hline Gastrointestinal bleeding, n (\%) & $1(1.3 \%)$ & $3(0.4 \%)$ & 0.323 & $1(1.7 \%)$ & $1(1.7 \%)$ & 1.000 \\
\hline Pain duration,d & $2.5 \pm 1.1$ & $1.8 \pm 1.2$ & 0.117 & $2.5 \pm 1.1$ & $2.9 \pm 1.3$ & 0.247 \\
\hline NOP duration, d & $2.9 \pm 1.0$ & $2.1 \pm 0.9$ & 0.122 & $2.9 \pm 1.1$ & $3.3 \pm 2.1$ & 0.142 \\
\hline ICU LOS, d & $3.0 \pm 1.9$ & $1.2 \pm 0.7$ & $<0.001^{*}$ & $3.0 \pm 1.8$ & $3.4 \pm 2.2$ & 0.067 \\
\hline Hospital LOS, d & $12.7 \pm 5.4$ & $10.3 \pm 5.1$ & 0.141 & $12.9 \pm 5.5$ & $16.3 \pm 7.7$ & $0.047^{*}$ \\
\hline Hospital costs, CNY & $26,517.2 \pm 2832.6$ & $18,246.2 \pm 5146.5$ & $<0.001^{*}$ & $25,348.4 \pm 2512.6$ & $32,421.7 \pm 2813.3$ & $0.002^{*}$ \\
\hline
\end{tabular}

Abbreviation: APD, abdominal paracentesis drainage; PCD, percutaneous catheter drainage; NOP, nil per os; LOS, length of stay

*Significant difference

LOS in the hospital were higher in the GC group than in the NGC group, but there were no significant differences $(P>0.05)$. The patients in the GC group had a higher hospital cost $(26,517.2 \pm 2832.6$ vs. $18,246.2 \pm 5146.5$, $P<0.001)$. These results also implied that the severity of the disease may have been higher in the GC group than in the NGC group.

After matching, the patients' clinical outcomes were reversed. The proportion of patients with organ failure ( $40.7 \%$ vs. $52.5 \%, P=0.041<0.05)$ and multi-organ failure (35.0\% vs. $67.7 \%, P=0.002<0.05)$ was significantly lower in the GC group than in the NGC group. The proportion of SAP was lower in the GC group than in the NGC group, although there was not a significant difference $(39.0 \%$ vs. $55.9 \%, P=0.065)$. Meanwhile, mortality in the GC group was also lower than that in the NGC group, although there was not a significant difference $(5.1 \%$ vs. $13.6 \%, P=0.113)$. There was no significant difference in LOS in the ICU $(3.0 \pm 1.8$ vs. $3.4 \pm 2.2, P=0.067)$ and proportion of patients using antibiotics $(47.5 \%$ vs.
49.2\%, $P=0.854$ ) between the two groups. Patients in the GC group had significantly lower LOS in the hospital $(12.9 \pm 5.5$ vs. $16.3 \pm 7.7, P=0.047<0.05)$ and cost $(25,348.4 \pm 2512.6$ vs. $32,421.7 \pm 2813.3, P=0.002<0.05)$ than those in the NGC group. At the same time, we found that the use of GCs did not increase the risk of gastrointestinal bleeding.

\section{GCs administration may facilitate fluid resuscitation in the early stage of AP}

We further analyzed fluid resuscitation in the early stage of AP (within $72 \mathrm{~h}$ ) in both groups (shown in Table 3). Before propensity score matching, patients in the GC group had significantly higher rehydration in the first $8 \mathrm{~h}$ than those in the NGC group $(2814.8 \pm 428.1$ vs. $1533.4 \pm 297.4, P<0.001)$. Compared to the NGC group, patients in the GC group showed a positive balance of fluid intake and output over $72 \mathrm{~h}$. After propensity score matching, there was no significant difference in rehydration volume in the 
Table 3 Fluid resuscitation-related characteristics

\begin{tabular}{|c|c|c|c|c|c|c|}
\hline \multirow[t]{2}{*}{ Characteristic } & \multicolumn{2}{|c|}{ Original cohort $(n=818)$} & \multirow[t]{2}{*}{$P$} & \multicolumn{2}{|c|}{ Matched cohort $(n=118)$} & \multirow[t]{2}{*}{$P$} \\
\hline & GC group $(n=76)$ & NGC group $(n=742)$ & & GC group $(n=59)$ & NGC group $(n=59)$ & \\
\hline $\begin{array}{l}\text { Amount of resuscitation flu- } \\
\text { ids within the first } 8 \mathrm{~h}, \mathrm{~mL}\end{array}$ & $2814.8 \pm 428.1$ & $1533.4 \pm 297.4$ & $<0.001^{*}$ & $2774.1 \pm 413.8$ & $2711.8 \pm 391.4$ & 0.947 \\
\hline \multicolumn{7}{|l|}{ Total fluid balance within } \\
\hline $8 \mathrm{~h}, \mathrm{~mL}$ & $2348.4 \pm 401.9$ & $1108.74 \pm 259.3$ & $<0.001^{*}$ & $2270.1 \pm 394.5$ & $2318.8 \pm 401.9$ & 0.884 \\
\hline $24 \mathrm{~h}, \mathrm{~mL}$ & $1798.1 \pm 394.8$ & $1342.9 \pm 377.4$ & $0.022^{*}$ & $1763.1 \pm 381.4$ & $1790.9 \pm 341.3$ & 0.941 \\
\hline $48 \mathrm{~h}, \mathrm{~mL}$ & $1371.8 \pm 331.4$ & $896.7 \pm 294.3$ & $0.039^{*}$ & $1355.6 \pm 324.7$ & $2057.1 \pm 313.1$ & $0.012^{*}$ \\
\hline $72 \mathrm{~h}, \mathrm{~mL}$ & $1159.1 \pm 284.2$ & $730.1 \pm 251.1$ & $0.007^{*}$ & $1189.4 \pm 267.4$ & $2545.1 \pm 338.7$ & $<0.001^{*}$ \\
\hline \multicolumn{7}{|c|}{ Hemodynamic and perfusion-related variables at $72 \mathrm{~h}$} \\
\hline Heart rate, /min & $113.1 \pm 10.9$ & $83.7 \pm 11.3$ & $<0.001^{*}$ & $110.9 \pm 10.7$ & $119.4 \pm 12.7$ & 0.374 \\
\hline $\mathrm{CVP}, \mathrm{cm} \mathrm{H} \mathrm{O}^{\#}$ & $11.8 \pm 2.1$ & $9.3 \pm 1.9$ & 0.127 & $11.7 \pm 2.1$ & $16.5 \pm 2.3$ & $<0.001^{*}$ \\
\hline Serum lactate, $\mathrm{mmol} / \mathrm{L}$ & $2.3 \pm 0.9$ & $1.2 \pm 0.5$ & 0.133 & $2.3 \pm 0.9$ & $2.7 \pm 1.1$ & 0.243 \\
\hline
\end{tabular}

*Significant difference

\# CVP, Central venous pressure. CVP was measured only in patients who had a central venous catheter. All the patients in the GC group ( $\mathrm{n}=76$ ) had measured the CVP. In NGC group, not all patients measured CVP $(n=314$, before matching; $n=57$, after matching)

first $8 \mathrm{~h}$ between the two groups $(2774.1 \pm 413.8$ vs. $2711.8 \pm 391.4, P=0.947)$. Patients in the NGC group showed a positive balance of fluid intake and output over $72 \mathrm{~h}$. Before matching, patients in the GC group had a significantly lower heart rate at $72 \mathrm{~h}$ than those in the NGC group $(113.1 \pm 10.9$ vs. $83.7 \pm 11.3$, $P<0.001)$. After matching, the heart rate was no significant difference between the two groups (110.9 \pm 10.7 vs. $119.4 \pm 12.7, P=0.374$ ) and the patients in the $\mathrm{GC}$ group had a significantly lower central venous pressure than those in the NGC group $(11.7 \pm 2.1$ vs. $16.5 \pm 2.3$, $P<0.001)$. However, central venous pressure was not measured in all patients. These results preferentially suggest that GCs administration may facilitate fluid resuscitation in the early stage of AP.
We analyzed CRP and TNF- $\alpha$ levels at admission, 3 days after admission, and 7 days after admission in matched patients (shown in Fig. 1). We found that CRP increased after admission in both groups. CRP was highest 3 days after admission and decreased 7 days after admission. Compared with that in the NGC group, the CRP in the GC group was more significantly lower 7 days after admission (139.1 \pm 53.2 vs. $188.5 \pm 59.4, P=0.013<0.05)$. In addition, TNF- $\alpha$ decreased after admission in both groups. TNF- $\alpha$ in the GC group also decreased more significantly 7 days after admission than in the NGC group (33.1 \pm 10.1 vs. $55.7 \pm 15.7, P=0.027<0.05)$.
A

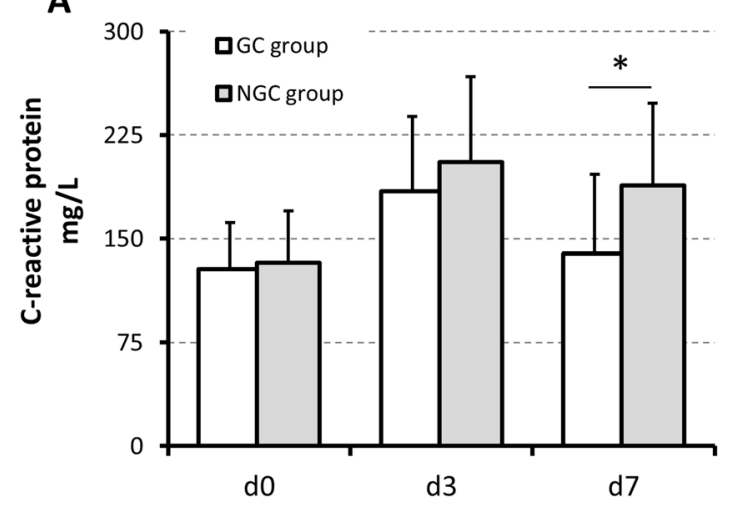

B

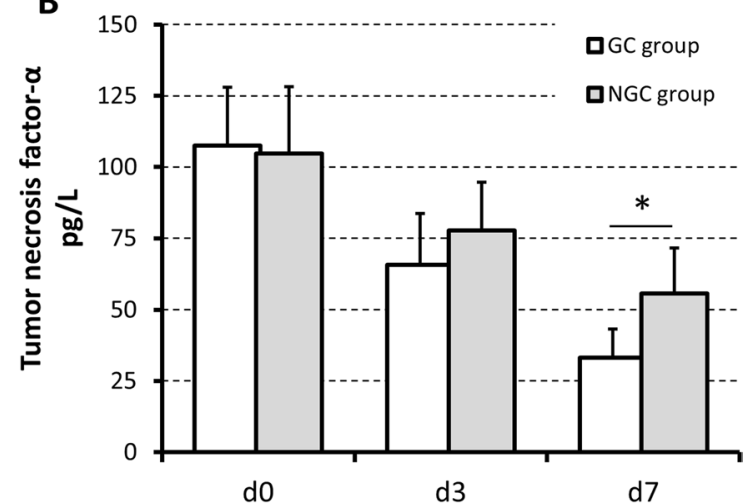

Fig. 1 Serum levels of C-reactive protein and Tumor necrosis factor-a in the GC and NGC groups 


\section{Discussion}

This retrospective research confirms that patients with AP can benefit from the utilization of GCs at the early phase of AP. The mortality, prevalence of multiorgan failure, hospital LOS, and costs were significantly reduced without increasing the incidence of gastrointestinal bleeding.

The role of GCs in the treatment of AP has been studied for a long time [15]. They have been considered a routine treatment for autoimmune pancreatitis [24]. However, the effects of GCs in the treatment of AP are controversial. On the one hand, to the best of our knowledge, Stephenson et al. first reported the benefit of GCs in human haemorrhagic AP in 1952 [14]. Several supporting case reports and clinical studies have been reported since then. For example, a recent study indicated that dexamethasone combined with Chinese herbal decoction decreases the risk of developing ARDS in patients with SAP [25], and a meta-analysis including six Chinese trials showed that corticosteroids might improve outcomes in patients with SAP [26]. GCs also show promising therapeutic effects in many animal models of AP, including ethionine-induced pancreatitis in rabbits, diet-induced necrotizing pancreatitis, and necrohaemorrhagic pancreatitis induced by the retrograde injection of $\mathrm{Na}$-taurocholate into the pancreatic duct in mice, canines, and porcine[16, 17, 27-30]. On the other hand, there are several randomized controlled trials, and a meta-analysis showed no statistically significant benefit of prophylactic GCs for the prevention of post-endoscopic retrograde cholangiopancreatography (ERCP) pancreatitis, one sort of AP [31]. Meanwhile, GCs are also thought to potentially trigger AP [32].

In our opinion, the effect of GCs in the treatment of AP needs to be re-evaluated. More quality studies are required to investigate whether GCs are beneficial in the treatment of AP.

First, the core pathophysiological process in the early stages of AP is uncontrolled inflammation [4]. Activation of inflammatory cascades induces a systemic inflammatory response and impairs organ functions [33]. GCs are almost the most widely prescribed anti-inflammatory drugs [34]. GCs play an anti-inflammatory role through induction of the synthesis of anti-inflammatory proteins and through repression of proinflammatory transcription factors, such as NF-kB [35], which are also activated during the early stages of AP [36].

GCs are beneficial in a variety of inflammatory diseases. For example, a recent meta-analysis showed that methylprednisolone treatment could accelerate the resolution of ARDS, which was also common in SAP, improving a broad spectrum of interrelated clinical outcomes [11]. In vivo, dexamethasone also showed a protective effect on acute kidney injury by preventing microvascular endothelial glycocalyx degradation initiated by TNF- $\alpha$ during SAP [27]. Furthermore, GCs have been shown to exhibit protective effects on systemic inflammatory responses induced by burns, infections, or even COVID$19[10,13]$. Thus, theoretically, GCs have a potential therapeutic effect on AP.

Second, a study showed that cyclooxygenase-2 (COX2) inhibitors can prevent AP from becoming severe recently [37]. This is the first report to demonstrate the effect of COX-2 inhibitors on the treatment of AP in humans. COX-2 inhibitors exert inflammatory inhibitory effects by blocking the production of prostaglandins from arachidonic acids [38]. COX-2 is a highly inducible enzyme and accumulates promptly in the presence of inflammation [39]. The anti-inflammatory properties of GCs are attributed in part to their interference with prostaglandin synthesis through cyclooxygenase [40]. This evidence also implies a possible role for GCs in the treatment of AP.

Third, the evidence that GCs have no prophylactic effect in post-ERCP pancreatitis does not disclaim that GCs have no therapeutic effect in AP. The occurrence of post-ERCP pancreatitis is closely associated with repeated intubation, intubation into the pancreatic duct, and longer ERCP procedures [41]. All of these factors are physically related to pancreatic duct insult or hypertension during the process of ERCP. Placement of pancreatic ductal stents after ERCP has also been shown to be effective in reducing the incidence of post-ERCP pancreatitis [42]. ERCP is also the standard treatment for pancreatitis due to biliary tract disease. Prophylactic GCs may not alleviate pancreatic duct insult or hypertension. Moreover, post-ERCP pancreatitis is usually mild or moderately severe and presents as a self-limiting process. The therapeutic effect of GCs in post-ERCP pancreatitis remains little studied until now.

Fourth, intravenous fluid resuscitation is considered the cornerstone of management in AP [43]. It is generally recommended in all patients with AP, despite the optimal rate, type, and goal of fluid resuscitation being controversial [44]. In a variety of inflammatory diseases, inflammatory cascades are thought to induce endothelial activation and capillary leakage, leading to circulatory collapse and shock [45]. GCs could preserve endothelial integrity through upregulation of junctional proteins such as occludin, claudin-5, and VE-cadherin and downregulation of matrix metalloproteinase-9 and alleviate hemodynamic disturbances [46, 47]. For example, low-dose GCs are considered to be associated with a faster reversal of shock and a short duration of mechanical ventilation in septic shock [48]. In patients undergoing cardiac surgery, GCs also reduce 
proinflammatory cytokine release, slow leukocyte migration, and decrease capillary leakage associated with cardiopulmonary bypass [49]. Moreover, in vivo, GCs have been shown to protect the renal microvascular endothelium and intestinal capillary endothelium during SAP [27]. These studies hint that GCs may fulfil a similar effect in the fluid resuscitation of AP.

However, GC-induced pancreatitis has only been reported in individual cases $[32,50]$. There is a lack of large-scale, high-quality evidence studies, and the mechanism of GC-induced pancreatitis remains unclear. GC-induced pancreatitis is currently thought to be correlated with high doses and long-term use and may be idiopathic [51].

The adverse effects of GCs are still noteworthy. Secondary infections, osteoporosis, wound healing difficulty, and gastrointestinal bleeding are the most concerning adverse effects of GCs therapy. GCsinduced immunosuppression is the predominant cause of secondary infections [52]. The utilization of a high dose of GCs could induce osteoporosis and eventually induce femoral head necrosis [53]. There is evidence supporting that using higher-dose GCs was an independent risk factor for bleeding [54]. Thus, patients with AP at low risk of infection were included in this study. Studies have confirmed that the risk of infection is low in the early stages of hypertriglyceridemic AP and that prophylactic antibiotic use is avoidable [55].

In this study, our results showed that the administration of GCs during the treatment of AP facilitated fluid resuscitation and reduced mortality, the prevalence of multi-organ failure, and hospital costs. At the same time, the administration of GCs during the early stage of AP did not increase the risk of infection or gastrointestinal bleeding. These data provide preliminary evidence that early GCs use is effective and relatively safe in the treatment of AP.

However, this study is retrospective, and there were some missing case details. Even though we used PSM analysis, inconsistencies in the baseline and bias could still be possible. In addition, in this study, the criterion was whether GCs should be used in the treatment of AP. We did not distinguish well between the types or amounts of GCs use or the duration of GCs use, which might also influence the therapeutic outcomes.

In summary, this retrospective study presents preliminary confirmation of the beneficial effect of GCs in the treatment of AP. More high-quality prospective studies are needed in the future.

Acknowledgements

Not applicable.

\section{Authors' contributions}

All authors have read and approved this manuscript. WM and JZX contributed equally to this work. WM and LHY designed the study and wrote the main manuscript text. WM and JZX conducted data collection and analysis. LHY made the final review of the article. All authors read and approved the final manuscript.

\section{Funding}

This research did not receive any specific grant from funding agencies in the public, commercial, or not-for-profit sectors.

\section{Availability of data and materials}

All datasets used and analyzed during the current study are available from the corresponding author upon reasonable request.

\section{Declarations}

Ethics approval and consent to participate

This study was approved by the Ethics Committee of the General Hospital of Western Theater Command and conducted in accordance with the Declaration of Helsinki. All methods were carried out in accordance with relevant guidelines and regulations. All patients signed the informed consent form.

Consent for publication

Not applicable.

\section{Competing interests}

The authors declare that they have no competing interests.

\section{Author details}

${ }^{1}$ Department of Traditional Chinese Medicine, General Hospital of Western Theater Command (Chengdu Military General Hospital), Chengdu, China. ${ }^{2}$ Department of General Surgery, General Hospital of Western Theater Command (Chengdu Military General Hospital), Chengdu 613000, China.

Received: 12 April 2021 Accepted: 14 August 2021

Published online: 25 August 2021

References

1. Banks PA, Bollen TL, Dervenis C, Gooszen HG, Johnson CD, Sarr MG, Tsiotos GG, Vege SS. Classification of acute pancreatitis-2012: revision of the Atlanta classification and definitions by international consensus. Gut. 2013;62(1):102-11.

2. Shah AP, Mourad MM, Bramhall SR. Acute pancreatitis: current perspectives on diagnosis and management. J Inflamm Res. 2018;11:77-85.

3. Bhatia M. Acute pancreatitis as a model of SIRS. Front Biosci (Landmark Ed). 2009;14:2042-50

4. Schepers NJ, Besselink MG, van Santvoort HC, Bakker OJ, Bruno MJ. Early management of acute pancreatitis. Best Pract Res Clin Gastroenterol. 2013;27(5):727-43.

5. Alam MM, Okazaki K, Nguyen LTT, Ota N, Kitamura H, Murakami S, Shima H, Igarashi K, Sekine H, Motohashi H. Glucocorticoid receptor signaling represses the antioxidant response by inhibiting histone acetylation mediated by the transcriptional activator NRF2. J Biol Chem. 2017:292(18):7519-30.

6. Huscher D, Thiele K, Gromnica-Ihle E, Hein G, Demary W, Dreher R, Zink A, Buttgereit F. Dose-related patterns of glucocorticoid-induced side effects. Ann Rheum Dis. 2009;68(7):1119-24

7. Chan ED, Chan MM, Chan MM, Marik PE. Use of glucocorticoids in the critical care setting: Science and clinical evidence. Pharmacol Ther. 2020;206:107428.

8. Fang F, Zhang Y, Tang J, Lunsford LD, Li T, Tang R, He J, Xu P, Faramand A, $\mathrm{Xu} \mathrm{J}$, et al. Association of corticosteroid treatment with outcomes in adult patients with sepsis: a systematic review and meta-analysis. JAMA Intern Med. 2019;179(2):213-23.

9. Huang J, Guo J, Li H, Huang W, Zhang T. Efficacy and safety of adjunctive corticosteroids therapy for patients with severe community-acquired 
pneumonia: A systematic review and meta-analysis. Medicine. 2019;98(13):e14636.

10. de Leeuw K, Niemeijer AS, Eshuis J, Nieuwenhuis MK, Beerthuizen Gl, Janssen WM. Effect and mechanism of hydrocortisone on organ function in patients with severe burns. J Crit Care. 2016:36:200-6.

11. Meduri GU, Bridges L, Shih MC, Marik PE, Siemieniuk RAC, Kocak M. Prolonged glucocorticoid treatment is associated with improved ARDS outcomes: analysis of individual patients' data from four randomized trials and trial-level meta-analysis of the updated literature. Intensive Care Med. 2016;42(5):829-40.

12. Ledford $\mathrm{H}$. Coronavirus breakthrough: dexamethasone is first drug shown to save lives. Nature. 2020;582(7813):469.

13. Wang K, Tan F, Zhou R, Liu D, Ni Z, Liu J, Luo F. Therapeutic response to corticosteroids in a critically ill patient with COVID-19: A case report. Medicine. 2020;99(31):e21597.

14. Stephenson HE Jr, Pfeffer RB, Saypol GM. Acute hemorrhagic pancreatitis; report of a case with cortisone treatment. AMA Arch Surg. 1952;65(2):307-8.

15. Shimosegawa T. Are glucocorticoids really useful for the treatment of acute pancreatitis? J Gastroenterol. 2002;37(7):580-1.

16. Studley JG, Schenk WG Jr. Pathophysiology of acute pancreatitis: evaluation of the effect and mode of action of steroids in experimental pancreatitis in dogs. Am J Surg. 1982;143(6):761-4.

17. Zhang XP, Zhang L, Wang Y, Cheng QH, Wang JM, Cai W, Shen HP, Cai J. Study of the protective effects of dexamethasone on multiple organ injury in rats with severe acute pancreatitis. JOP: J Pancreas. 2007:8(4):400-12.

18. Valdivielso P, Ramírez-Bueno A, Ewald N. Current knowledge of hypertriglyceridemic pancreatitis. Eur J Intern Med. 2014;25(8):689-94.

19. Larvin M, McMahon MJ. APACHE-II score for assessment and monitoring of acute pancreatitis. Lancet (London, England). 1989:2(8656):201-5.

20. Vincent JL, Moreno R, Takala J, Willatts S, De Mendonça A, Bruining H, Reinhart CK, Suter PM, Thijs LG. The SOFA (Sepsis-related Organ Failure Assessment) score to describe organ dysfunction/failure. On behalf of the Working Group on Sepsis-Related Problems of the European Society of Intensive Care Medicine. Intensive Care Med. 1996;22(7):707-10.

21. Tenner S, Baillie J, DeWitt J, Vege SS. American College of Gastroenterology guideline: management of acute pancreatitis. Am J Gastroenterol. 2013;108(9):1400-15.

22. Crockett SD, Wani S, Gardner TB, Falck-Ytter Y, Barkun AN. American Gastroenterological Association Institute Guideline on initial management of acute pancreatitis. Gastroenterology. 2018;154(4):1096-101.

23. Liu WH, Ren LN, Chen T, Liu LY, Jiang JH, Wang T, Xu C, Yan HT, Zheng XB, Song $\mathrm{FQ}$, et al. Abdominal paracentesis drainage ahead of percutaneous catheter drainage benefits patients attacked by acute pancreatitis with fluid collections: a retrospective clinical cohort study. Crit Care Med. 2015:43(1):109-19.

24. Cai O, Tan S. From pathogenesis, clinical manifestation, and diagnosis to treatment: an overview on autoimmune pancreatitis. Gastroenterol Res Pract. 2017;2017:3246459.

25. Wan MH, Li J, Gong HL, Xue P, Zhu L, Chen GY, Xia Q, Wen-Fu T. Clinical observation on the effect of dexamethasone and Chinese herbal decoction for purgation in severe acute pancreatitis patients. Chin J Integr Med. 2011;17(2):141-5.

26. Dong LH, Liu ZM, Wang SJ, Zhao SJ, Zhang D, Chen Y, Wang YS. Corticosteroid therapy for severe acute pancreatitis: a meta-analysis of randomized, controlled trials. Int J Clin Exp Pathol. 2015:8(7):7654-60.

27. Yu WQ, Zhang SY, Fu SQ, Fu QH, Lu WN, Zhang J, Liang ZY, Zhang Y, Liang TB. Dexamethasone protects the glycocalyx on the kidney microvascular endothelium during severe acute pancreatitis. J Zhejiang Univ Sci B. 2019;20(4):355-62.

28. Okanishi H, Nagata T, Nakane S, Watari T. Comparison of initial treatment with and without corticosteroids for suspected acute pancreatitis in dogs. J Small Anim Pract. 2019;60(5):298-304.

29. Zhao S, Yang J, Liu T, Zeng J, Mi L, Xiang K. Dexamethasone inhibits NF-кBp65 and HMGB1 expression in the pancreas of rats with severe acute pancreatitis. Mol Med Rep. 2018;18(6):5345-52.

30. Yu M, Yang Z, Zhu Y, Lu N. Efficacy of glucocorticoids in rodents of severe acute pancreatitis: a meta-analysis. Int J Clin Exp Pathol. 2014;7(7):3647-61.
31. Zheng M, Bai J, Yuan B, Lin F, You J, Lu M, Gong Y, Chen Y. Meta-analysis of prophylactic corticosteroid use in post-ERCP pancreatitis. BMC Gastroenterol. 2008:8:6.

32. Yahiaoui N, Roche M, Aissaoui-Hoffmann N, Keita BA, Mallaret M. Intravenous methylprednisolone induced acute pancreatitis. Eur J Clin Pharmacol. 2017;73(5):645-6.

33. Balk RA. Systemic inflammatory response syndrome (SIRS): where did it come from and is it still relevant today? Virulence. 2014;5(1):20-6.

34. Syed AP, Greulich F, Ansari SA, Uhlenhaut NH. Anti-inflammatory glucocorticoid action: genomic insights and emerging concepts. Curr Opin Pharmacol. 2020;53:35-44.

35. Smoak KA, Cidlowski JA. Mechanisms of glucocorticoid receptor signaling during inflammation. Mech Ageing Dev. 2004;125(10-11):697-706.

36. Huang H, Liu Y, Daniluk J, Gaiser S, Chu J, Wang H, Li ZS, Logsdon CD, Ji $B$. Activation of nuclear factor- $K B$ in acinar cells increases the severity of pancreatitis in mice. Gastroenterology. 2013;144(1):202-10.

37. Huang Z, Ma X, Jia X, Wang R, Liu L, Zhang M, Wan X, Tang C, Huang L. Prevention of severe acute pancreatitis with cyclooxygenase-2 inhibitors: a randomized controlled clinical trial. Am J Gastroenterol. 2020;115(3):473-80.

38. Bindu S, Mazumder S, Bandyopadhyay U. Non-steroidal anti-inflammatory drugs (NSAIDs) and organ damage: a current perspective. Biochem Pharmacol. 2020;180:114147.

39. Dhingra AK, Chopra B, Dass R, Mittal SK. An update on anti-inflammatory compounds: a review. Anti-Inflamm Antiallergy Agents Med Chem. 2015;14(2):81-97.

40. Dequeker J. NSAIDs/corticosteroids-primum non nocere. Adv Exp Med Biol. 1999;455:319-25

41. Kochar B, Akshintala VS, Afghani E, Elmunzer BJ, Kim KJ, Lennon AM Khashab MA, Kalloo AN, Singh VK. Incidence, severity, and mortality of post-ERCP pancreatitis: a systematic review by using randomized, controlled trials. Gastrointest Endosc. 2015;81(1):143-149.e149.

42. Choudhary A, Bechtold ML, Arif M, Szary NM, Puli SR, Othman MO, Pais WP, Antillon MR, Roy PK. Pancreatic stents for prophylaxis against postERCP pancreatitis: a meta-analysis and systematic review. Gastrointest Endosc. 2011;73(2):275-82

43. Trikudanathan G, Navaneethan U, Vege SS. Current controversies in fluid resuscitation in acute pancreatitis: a systematic review. Pancreas. 2012;41(6):827-34.

44. Vege SS, DiMagno MJ, Forsmark CE, Martel M, Barkun AN. Initial medical treatment of acute pancreatitis: american gastroenterological association institute technical review. Gastroenterology. 2018;154(4):1103-39.

45. Zielińska KA, Van Moortel L, Opdenakker G, De Bosscher K, Van den Steen PE. Endothelial response to glucocorticoids in inflammatory diseases. Front Immunol. 2016;7:592.

46. Kashiwamura Y, Sano Y, Abe M, Shimizu F, Haruki H, Maeda T, Kawai M Kanda T. Hydrocortisone enhances the function of the blood-nerve barrier through the up-regulation of claudin-5. Neurochem Res. 2011;36(5):849-55.

47. Cui N, Wang H, Long Y, Su L, Liu D. Dexamethasone suppressed LPSinduced matrix metalloproteinase and its effect on endothelial glycocalyx shedding. Mediat Inflamm. 2015;2015:912726.

48. Oppert M, Schindler R, Husung C, Offermann K, Gräf KJ, Boenisch O, Barckow D, Frei U, Eckardt KU. Low-dose hydrocortisone improves shock reversal and reduces cytokine levels in early hyperdynamic septic shock. Crit Care Med. 2005;33(11):2457-64.

49. Taleska Stupica G, Sostaric M, Bozhinovska M, Rupert L, Bosnic Z, Jerin A, Ihan A, Klokocovnik T, Podbregar M. Extracorporeal hemadsorption versus glucocorticoids during cardiopulmonary bypass: a prospective, randomized, controlled trial. Cardiovasc Ther. 2020;2020:7834173.

50. Yoshizawa Y, Ogasa S, Izaki S, Kitamura K. Corticosteroid-induced pancreatitis in patients with autoimmune bullous disease: case report and prospective study. Dermatology (Basel, Switzerland). 1999;198(3):304-6.

51. Nango D, Hirose $Y$, Goto M, Echizen H. Analysis of the Association of Administration of various glucocorticoids with development of acute pancreatitis using US Food and Drug Administration adverse event reporting system (FAERS). J Pharm Health Care Sci. 2019;5:5.

52. Schoenle LA, Moore IT, Dudek AM, Garcia EB, Mays M, Haussmann MF, Cimini D, Bonier F. Exogenous glucocorticoids amplify the costs of infection by reducing resistance and tolerance, but effects are mitigated by co-infection. Proc Biol Sci. 1900;2019(286):20182913. 
53. Chang C, Greenspan A, Gershwin ME. The pathogenesis, diagnosis and clinical manifestations of steroid-induced osteonecrosis. J Autoimmun. 2020;110:102460.

54. Kondo Y, Hatta W, Koike T, Takahashi Y, Saito M, Kanno T, Asanuma K, Asano N, Imatani A, Masamune A. The use of higher dose steroids increases the risk of rebleeding after endoscopic hemostasis for peptic ulcer bleeding. Dig Dis Sci. 2018;63(11):3033-40.

55. Párniczky A, Lantos T, Tóth EM, Szakács Z, Gódi S, Hágendorn R, Ilés D, Koncz B, Márta K, Mikó A, et al. Antibiotic therapy in acute pancreatitis: from global overuse to evidence based recommendations. Pancreatol: Off J Int Assoc Pancreatol (IAP) [et al]. 2019;19(4):488-99.

\section{Publisher's Note}

Springer Nature remains neutral with regard to jurisdictional claims in published maps and institutional affiliations.
Ready to submit your research? Choose BMC and benefit from:

- fast, convenient online submission

- thorough peer review by experienced researchers in your field

- rapid publication on acceptance

- support for research data, including large and complex data types

- gold Open Access which fosters wider collaboration and increased citations

- maximum visibility for your research: over $100 \mathrm{M}$ website views per year

At BMC, research is always in progress.

Learn more biomedcentral.com/submissions 